\title{
First results of the air shower experiment KASCADE
}

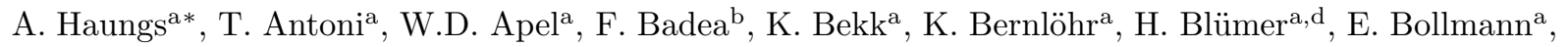 \\ H. Bozdog ${ }^{\text {b }}$ I.M. Brancus ${ }^{\mathrm{b}}$, C. Büttner ${ }^{\mathrm{a}}$, A. Chilingarian ${ }^{\mathrm{c}}$, K. Daumiller ${ }^{\mathrm{d}}$, P. Doll ${ }^{\mathrm{a}}$, J. Engler ${ }^{\mathrm{a}}$, \\ F. Feßler ${ }^{\text {a }}$, H.J. Gils ${ }^{\mathrm{a}}$, R. Glasstetter ${ }^{\mathrm{d}}$, R. Haeusler ${ }^{\mathrm{a}}$, W. Hafemann ${ }^{\mathrm{a}}$, D. Heck ${ }^{\mathrm{a}}$, J.R. Hörandel ${ }^{\mathrm{d} \dagger}$, \\ T. Holst ${ }^{\mathrm{a}}$, K.-H. Kampert ${ }^{\mathrm{a}, \mathrm{d}}$, H. Keim ${ }^{\mathrm{a}}$, J. Kempa ${ }^{\mathrm{e}}$, H.O. Klages ${ }^{\mathrm{a}}$, J. Knapp ${ }^{\mathrm{d} \ddagger}$, D. Martello ${ }^{\mathrm{d}}$, \\ H.J. Mathes ${ }^{\mathrm{a}}$, P. Matussek ${ }^{\mathrm{a}}$, H.J. Mayer ${ }^{\mathrm{a}}$, J. Milke ${ }^{\mathrm{a}}$, D. Mühlenberg ${ }^{\mathrm{a}}$, J. Oehlschläger ${ }^{\mathrm{a}}$, M. Petcu ${ }^{\mathrm{b}}$,

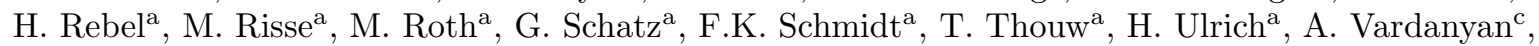 \\ B. Vulpescu ${ }^{\mathrm{b}}$, J.H. Weber ${ }^{\mathrm{a}}$, J. Wentz ${ }^{\mathrm{a}}$, T. Wiegert ${ }^{\mathrm{a}}$, J. Wochele ${ }^{\mathrm{a}}$, J. Zabierowski ${ }^{\mathrm{f}}$, S. Zagromski $^{\mathrm{a}}$

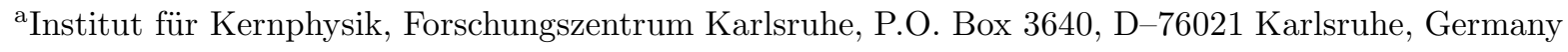 \\ ${ }^{\mathrm{b}}$ Institute of Physics and Nuclear Engineering, RO-7690 Bucharest, Romania \\ ${ }^{\mathrm{c} C o s m i c ~ R a y ~ D i v i s i o n, ~ Y e r e v a n ~ P h y s i c s ~ I n s t i t u t e, ~ Y e r e v a n ~ 36, ~ A r m e n i a ~}$ \\ ${ }^{\mathrm{d}}$ Institut für Experimentelle Kernphysik, Universität Karlsruhe, D-76021 Karlsruhe, Germany \\ e Department of Experimental Physics, University of Lodz, PL-90236 Lodz, Poland \\ ${ }^{\mathrm{f}}$ Soltan Institute for Nuclear Studies, PL-90950 Lodz, Poland
}

The main goals of the KASCADE (KArlsruhe Shower Core and Array DEtector) experiment are the determination of the energy spectrum and elemental composition of the charged cosmic rays in the energy range around the knee at $\approx 5 \mathrm{PeV}$. Due to the large number of measured observables per single shower a variety of different approaches are applied to the data, preferably on an event-by-event basis. First results are presented and the influence of the high-energy interaction models underlying the analyses is discussed.

\section{INTRODUCTION}

The air shower experiment KASCADE [1] aims at the investigation of the knee region of the charged cosmic rays. It is built up as a multidetector setup for measuring simultaneously a large number of observables in the different particle (electromagnetic, muonic and hadronic) components of the extended air shower (EAS). This enables to perform a multivariate multiparameter analysis for the registered EAS on an event-by-event basis to account for the non parametric, stochastic processes of the EAS development in the atmosphere. In parallel the KASCADE collaboration tries to improve the tools for the Monte Carlo simulations with the relevant physics. The code CORSIKA 22 allows not only the detailed three dimensional

\footnotetext{
*corresponding author, e-mail: haungs@ik3.fzk.de

${ }^{\dagger}$ now at: University of Chicago, Chicago, IL 60637

${ }^{\ddagger}$ now at: University of Leeds, Leeds LS2 9JT, U.K.
}

simulation of the shower development in all particle components (including neutrinos) down to the observation level, but it has been implemented several high-energy interaction models. As the basic physics of these models in the relevant energy region and in the extreme forward direction cannot be tested at present days' accelerators, the test of these models emerged as one of the goals of the KASCADE experiment. The following overview is based on results presented at the $26^{\text {th }}$ International Cosmic Ray Conference in Salt Lake City, Utah 1999 [3].

\section{THE KASCADE EXPERIMENT}

The KASCADE array consists of 252 detector stations in a $200 \times 200 \mathrm{~m}^{2}$ rectangular grid containing unshielded liquid scintillation detectors (e/ $\gamma$-detectors) and below $10 \mathrm{~cm}$ lead and $4 \mathrm{~cm}$ steel plastic scintillators as muon-detectors. The 
total sensitive areas are $490 \mathrm{~m}^{2}$ for the $e / \gamma$ - and $622 \mathrm{~m}^{2}$ for the muon-detectors. In the center of the array a hadron calorimeter $\left(16 \times 20 \mathrm{~m}^{2}\right)$ is built up, consisting of more than 40,000 liquid ionisation chambers in 8 layers with a trigger layer consisting of 456 scintillation detectors in between. Below the calorimeter a setup of position sensitive multiwire proportional chambers (MWPC) in two layers measures high-energy muons $\left(E_{\mu}>2 \mathrm{GeV}\right)$ of the EAS.

For each single shower a large number of observables are reconstructed with small uncertainties. For example, the errors for the so-called shower sizes, i.e. total numbers of electrons $N_{e}$ and number of muons in the range of the core distance $40-200 \mathrm{~m} N_{\mu}^{t r}$, are smaller than $10 \%$.

The resulting frequency spectra of the sizes (inclusive the spectra of the hadron number and muon density spectra at different core distances) show kinks at same integral fluxes. This is a strong hint for an astrophysical source of the knee phenomenon based on pure experimental data, since same intensity of the flux corresponds to equal primary energy.

But for the reconstruction of the primary energy spectrum and the chemical composition detailed Monte Carlo simulations are indispensable due to the unknown initial parameters and the large intrinsic fluctuations of the stochastic process of the shower development in the atmosphere. The usage of a larger number of less correlated observables in a multivariate analysis parallel to independent tests of the simulation models tries to find the solution of this dilemma.

\section{ANALYSES AND RESULTS}

In the air shower simulation program CORSIKA several high-energy interaction models are embedded including VENUS, QGSJET and SIBYLL (Refs. see in [2]). The models are based on the Gribov-Regge-theory and QCD in accordance with accelerator data. Extrapolations for the EAS physics in the knee region are necessary due to the high interaction energy and for the extreme forward direction. To compare KASCADE data with Monte Carlo expectations a detector simulation by GEANT is performed for each CORSIKA

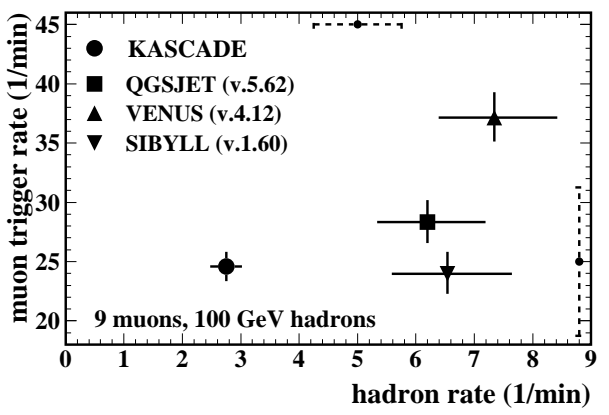

Figure 1. Comparison of simulated and measured integral muon trigger and hadron rates. Uncertainties of the elements' absolute fluxes of the relevant energy range is indicated by dotted lines.

simulated shower.

One test is the comparison of simulated integral muon trigger and hadron rates with the measurements. This test is sensitive to the energy spectrum of the hadrons which are produced in the forward direction at primary energies around 10 $\mathrm{TeV}$, where the chemical composition is roughly known (Fig.11). For higher primary energies the hadronic part of the interaction models are tested by comparisons of different hadronic observables in ranges of shower sizes [ $[$. In general it is seen that the high-energy interaction models predict a too large number of hadrons at sea level compared with the measurements.

Nonparametric multivariate methods like "Neural Networks" or analyses based on the "Bayesian decision rules" are applied to the KASCADE data for the estimation of the energy and mass of the cosmic rays on an event-by-event basis. The necessary "a-priori" information in form of probability density distributions are won by detailed Monte Carlo simulations with large statistics.

For the energy reconstruction the shower sizes $N_{e}$ and $N_{\mu}^{t r}$ as parameters are used in a neural network analyses (Fig.2). A parametric approach to the same data leads to compatible results (Fig.2): here a simultaneous fit to the $N_{e}$ and $N_{\mu}^{t r}$ size spectra is performed. The kernel function of this fit contains the size-energy correlations for two primary masses (proton and iron) obtained by Monte Carlo simulations. 


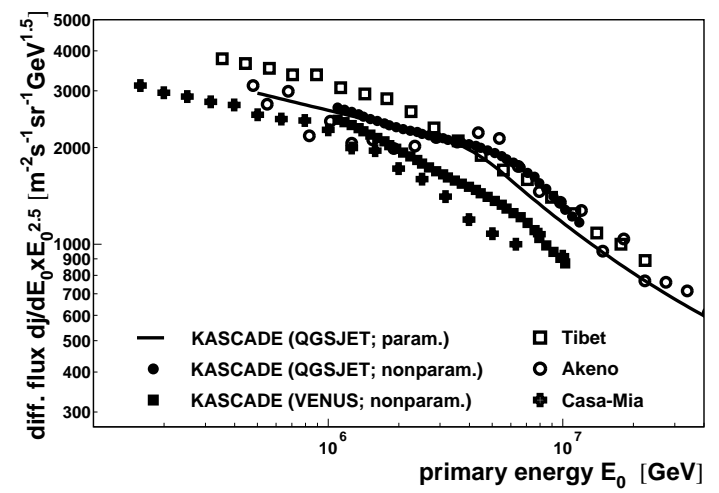

Figure 2. The primary cosmic ray energy spectrum from KASCADE and other experiments. The spectral index changes from $\approx-2.7$ to $\approx-3.1$ at the knee position of $\approx 5 \cdot 10^{6} \mathrm{GeV}$.

An analysis of the size-ratio $\lg \left(N_{\mu}^{t r}\right) / \lg \left(N_{e}\right)$ calculated for each single event leads to results of the elemental composition for different energy ranges (Fig.33). The measured distribution of these ratios is assumed to be a superposition of simulated distributions for different primary masses. The large iron sampling calorimeter of KASCADE allows to investigate the hadronic part of EAS in terms of the chemical composition. For six different hadronic observables (won by spatial and energy distributions of the hadrons) the deviations of the mean values to expectations of pure proton and iron primaries in certain energy ranges are calculated.

Besides the use of global parameters like the shower sizes, sets of different parameters are used for neural network and Bayesian decision analyses. Examples of such observables are the number of reconstructed hadrons in the calorimeter, their reconstructed energy sum, number of muons in the shower center, or parameters obtained by a fractal analysis of the hit pattern of muons and secondaries at the MWPC. The latter ones are sensitive to the structure of the shower core which is mass sensitive due to different shower developments of light and heavy particles in the atmosphere. In Figure 3 results of a Bayesian analyses and of a separate neural net analysis using the fractal parameters are shown.

As the tendency of the results of each described

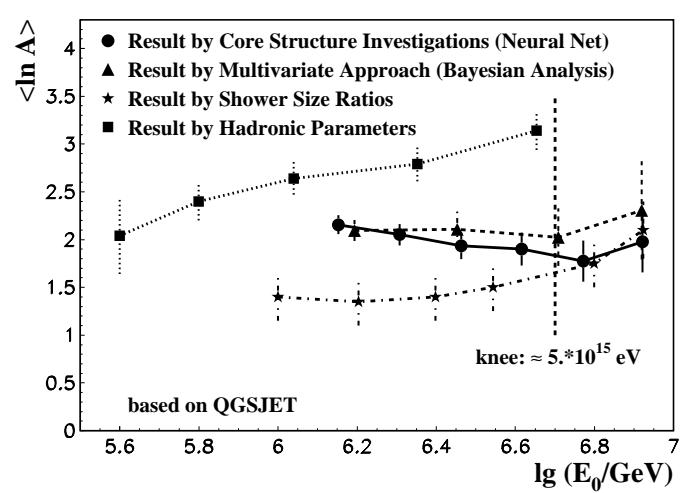

Figure 3. The chemical composition estimated with the KASCADE data, using different methods and observables from different particle components.

method is consistent with a heavier primary mass after the knee region, but the absolute scale strongly depends on the particle component of which the observables are constructed from, the syllogism is that the balance of the energy and number of particles between the muonic, electromagnetic and hadronic part in the EAS differs for simulations and the real shower development.

\section{CONCLUSIONS}

First results of the KASCADE experiment can be summarized by following statements: All secondary particle components of the showers display a kink in the size spectra. This strongly supports an astrophysical origin of the "knee", rather than effects of the interaction of the primaries in the atmosphere. The knee is sharper for the light primary component than for the heavy one. This result follows from the measurement as an increasing average mass of the primary cosmic rays above the observed kink, together with the energy dependent mass classification of single air showers. But none of the high-energy interaction models en vogue is able to fit the data of all observables consistently.

\section{REFERENCES}

1. H.O. Klages et al. - KASCADE collaboration, Nucl. Phys. B (Proc. Suppl.) 52B (1997) 
92.

2. D. Heck et al., FZKA 6019, Forschungszentrum Karlsruhe (1998).

3. T. Antoni et al. - KASCADE collaboration, Proc. $26^{\text {th }}$ ICRC, Salt Lake City, Utah 1999; published as: K.H. Kampert (Editor), FZKA 6345, Forschungszentrum Karlsruhe (1999).

4. T. Antoni et al. - KASCADE collaboration, J. Phys. G: Nucl. Part. Phys. 25 (1999) 2161. 